\title{
TU/e EmonONEN

\section{A novel approach to estimate trabecular bone anisotropy from stress tensors}

Citation for published version (APA):

Hazrati Marangalou, J., Ito, K., \& Rietbergen, van, B. (2015). A novel approach to estimate trabecular bone anisotropy from stress tensors. Biomechanics and Modeling in Mechanobiology, 14(1), 39-48.

https://doi.org/10.1007/s10237-014-0584-6

DOI:

10.1007/s10237-014-0584-6

Document status and date:

Published: 01/01/2015

\section{Document Version:}

Accepted manuscript including changes made at the peer-review stage

\section{Please check the document version of this publication:}

- A submitted manuscript is the version of the article upon submission and before peer-review. There can be important differences between the submitted version and the official published version of record. People interested in the research are advised to contact the author for the final version of the publication, or visit the $\mathrm{DOI}$ to the publisher's website.

- The final author version and the galley proof are versions of the publication after peer review.

- The final published version features the final layout of the paper including the volume, issue and page numbers.

Link to publication

\section{General rights}

Copyright and moral rights for the publications made accessible in the public portal are retained by the authors and/or other copyright owners and it is a condition of accessing publications that users recognise and abide by the legal requirements associated with these rights.

- Users may download and print one copy of any publication from the public portal for the purpose of private study or research.

- You may not further distribute the material or use it for any profit-making activity or commercial gain

- You may freely distribute the URL identifying the publication in the public portal.

If the publication is distributed under the terms of Article $25 \mathrm{fa}$ of the Dutch Copyright Act, indicated by the "Taverne" license above, please follow below link for the End User Agreement:

www.tue.nl/taverne

Take down policy

If you believe that this document breaches copyright please contact us at:

openaccess@tue.nl

providing details and we will investigate your claim. 
A novel approach to estimate trabecular bone anisotropy from stress tensors

\section{Javad Hazrati Marangalou, Keita Ito \& Bert van Rietbergen}

Biomechanics and Modeling in Mechanobiology

ISSN 1617-7959

Biomech Model Mechanobiol DOI 10.1007/s10237-014-0584-6

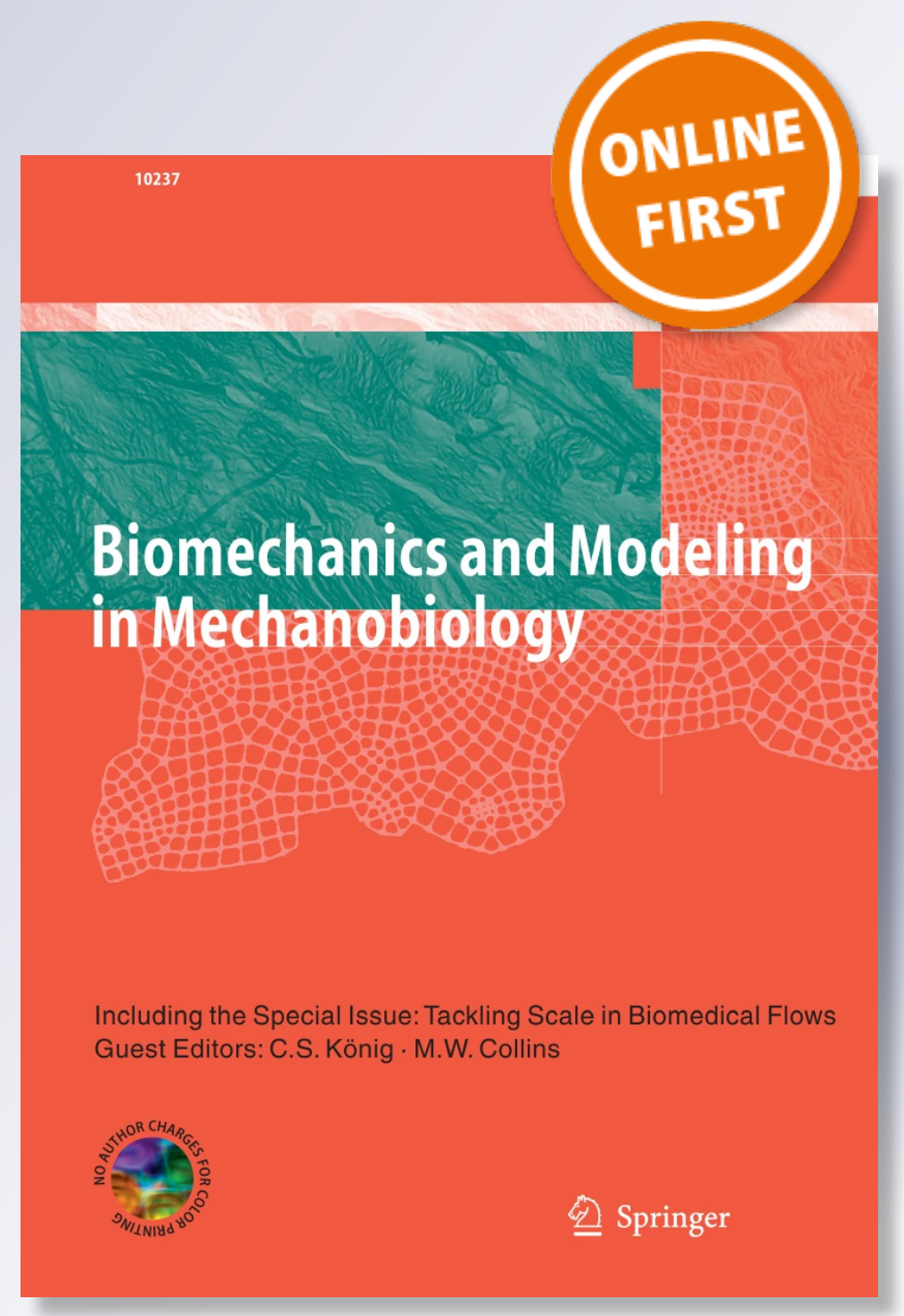

是 Springer 
Your article is protected by copyright and all rights are held exclusively by SpringerVerlag Berlin Heidelberg. This e-offprint is for personal use only and shall not be selfarchived in electronic repositories. If you wish to self-archive your article, please use the accepted manuscript version for posting on your own website. You may further deposit the accepted manuscript version in any repository, provided it is only made publicly available 12 months after official publication or later and provided acknowledgement is given to the original source of publication and a link is inserted to the published article on Springer's website. The link must be accompanied by the following text: "The final publication is available at link.springer.com". 


\title{
A novel approach to estimate trabecular bone anisotropy from stress tensors
}

\author{
Javad Hazrati Marangalou • Keita Ito • \\ Bert van Rietbergen
}

Received: 13 August 2013 / Accepted: 6 April 2014

(C) Springer-Verlag Berlin Heidelberg 2014

\begin{abstract}
Continuum finite element (FE) models of bones and bone-implant configurations are usually based on clinical CT scans. In virtually all of these models, material properties assigned to the bone elements are chosen as isotropic. It has been shown, however, that cancellous bone can be highly anisotropic and that its elastic behavior is best described as orthotropic. Material models have been proposed to derive the orthotropic elastic constants from measurements of density and a fabric tensor. The use of such relationships in FE models derived from CT scans, however, is hampered by the fact that the measurement of such a fabric tensor is not possible from clinical CT images since the resolution of such images is not good enough to resolve the trabecular micro-architecture. In this study, we explore an alternative approach that is based on the paradigm that bone adapts its micro-architecture to the loading conditions, hence that fabric and stress tensors should be aligned and correlated. With this approach, the eigenvectors and eigenvalues of the element continuum-level stress tensor are used as an estimate of the element fabric tensor, from which the orthotropic material properties then are derived. Using an iterative procedure, element orthotropic material properties and fabric tensors are updated until a converged situation is reached. The goals of this study were to investigate the feasibility and accuracy of such an iterative approach to derive orthotropic material properties for a human proximal femur and to investigate whether models derived in this way can provide more accurate results than isotropic models. Results were com-
\end{abstract}

J. Hazrati Marangalou $\cdot$ K. Ito · B. van Rietbergen ( $₫)$ Orthopaedic Biomechanics, Department of Biomedical Engineering, Eindhoven University of Technology, Eindhoven, The Netherlands e-mail: B.v.Rietbergen@tue.nl

J. Hazrati Marangalou

e-mail: J.Hazrati.Marangalou@tue.nl pared to those obtained from models of the same femurs for which the fabric was measured from micro-CT scans. It was found that the iterative approach could well estimate the orientation of the fabric principal directions. When comparing the stress/damage values in the models with material properties based on estimated and measured fabric tensors, the differences were not significant, suggesting that the material properties based on the estimated fabric tensor well reflected those based on the measured fabric tensor. Errors were less than those obtained when using isotropic models. It is concluded that this novel approach can provide a reasonable estimate of anisotropic material properties of cancellous bone. We expect that this approach can lead to more accurate results in particular for models used to study implants, which are usually anchored in highly anisotropic cancellous bone regions.

Keywords Anisotropy - Trabecular bone - Fabric tensor . Finite element analysis

\section{Introduction}

Continuum-level finite element (FE) models of bones are often used for preclinical evaluation of implants and orthopedic interventions. Such FE models are commonly generated from CT scans, such that a patient-specific geometry and density distribution can be assigned. The bone material properties are then derived using some sort of empirical power law that relates the measured density to local bone stiffness and strength (Carter and Hayes 1976; Keyak 2001; Morgan et al. 2003; Rice et al. 1988; Taddei et al. 2007). In such studies, stiffness and strength properties are usually modeled as isotropic. In particular in cancellous regions, however, bone can be highly anisotropic due to its structural organization 
of trabeculae in preferred directions, as commonly quantified by a so-called fabric tensor (Cowin 1985; Harrigan and Mann 1984; Odgaard 1997; Zysset et al. 1998). Since the resolution of clinical CT imaging techniques is not sufficient to capture this organization, it is not possible to account for this anisotropic behavior in continuum FE models.

In an earlier study, we introduced an approach that makes use of a database of high-resolution micro-CT scans from which the fabric can be obtained, which is then mapped to a patient model. In this way, it is possible to create FE models with patient-specific geometry and density and databasederived anisotropy. In that study, we also demonstrated that such models better reproduce the stress and damage distribution as well as bone stiffness and strength than isotropic models (Hazrati Marangalou et al. 2013). A disadvantage of this approach, however, is that it can be used only for sites for which such databases are available.

In the present study, we explore a more versatile alternative approach that does not require a database for deriving anisotropic material properties in continuum models of bone. This alternative approach is based on the commonly accepted paradigm that bone adapts its micro-architecture to the loading conditions. The fact that elastic properties of cancellous bone are best described as orthotropic (Pahr and Zysset 2009; Yang et al. 1998) has been explained by the fact that its underlying micro-architecture is adapted to a second-rank continuum-level stress tensor (Odgaard et al. 1997; Turner 1992). With this adaptation, a bone microarchitecture is formed in which most trabeculae are oriented in the direction where, on average, the magnitude of the principal stress is maximal, and less trabeculae are oriented in the direction where, on average, the magnitude of the principal stress is minimal (Fyhrie and Carter 1986; Vander Sloten and Van der Perre 1989). As a result of this adaptive process, the orientation of the orthotropic axes will thus be expected to coincide with the principal stress directions, whereas the degree of anisotropy relates to the degree of anisotropy of the principal stress magnitudes (Turner 1992). Earlier bone remodeling simulations have demonstrated that predictions of bone density and anisotropy indeed can produce realistic results in 2D models of the femur (Jacobs et al. 1997). This suggests that it would be possible as well to derive the fabric tensor from measurements of the principal stress orientation and magnitudes, such as can be obtained from continuum FE analyses. This, however, would be possible only if the correct anisotropic material properties are specified in the FE model, which is not known beforehand. A solution to this problem that is investigated in this study is to use an iterative approach that starts with isotropic material properties to calculate the principal stresses from which orthotropic material properties are calculated. Using these updated orthotropic material properties, the principal stresses are re-evaluated and the material properties updated accord- ingly. This procedure can be repeated until no more changes are found.

The first goal of this study was to investigate the feasibility and accuracy of such an iterative approach to calculate the fabric tensor from the stress tensor. In order to investigate the accuracy, the approach was applied using continuum FE models of 10 femurs that were generated from micro-CT images, which made it possible to compare the estimated fabric tensors from stress tensors with the ones measured from the bone micro-architecture. The data set used was the same as the one used in the earlier study where we tested the database approach to derive anisotropic properties (Hazrati Marangalou et al. 2013). By comparing our present results with results obtained in that study, our second goal was to investigate whether this alternative approach can provide more accurate results than models generated by a database approach or isotropic models which account only for the density distribution.

\section{Materials and methods}

\subsection{Materials}

Ten human cadaver femurs (mean age: $71.9 \pm 8.55$ years) obtained from three female and seven male donors were used for this study. The femurs were selected from a database of 33 femurs used in earlier studies (Hazrati Marangalou et al. 2013, 2014b). The selected femurs are the same as the ones used in an earlier study that uses a database approach to derive anisotropic properties, in order to facilitate an easy comparison between results of that earlier approach and the present approach. Imaging and processing of these femurs was described earlier (Hazrati Marangalou et al. 2014b). In summary, micro-CT scans (XtremeCT, Scanco Medical AG, Brüttisellen, Switzerland) of the most proximal part (9-12 cm in length) were made with a nominal isotropic resolution of $82 \mu \mathrm{m}$. Images were filtered and processed according to the protocol recommended by the manufacturer. Compartments of cortical and cancellous bone were identified using masks obtained from a filtering procedure, while also all bone within $1 \mathrm{~mm}$ from the periosteal surface was identified as part of the cortical compartment.

Continuum finite element models of the proximal femurs were generated based on contours of the bone periosteal surface as obtained using software provided with the microCT scanner (IPL V5.16, Scanco Medical AG, Brüttisellen, Switzerland). Using a marching cube algorithm, a triangularization of the bone surface was made and exported in STL format to ANSYS finite element software (Ansys Inc., USA) for volumetric meshing. Meshing was done using secondorder tetrahedron elements with a typical size of $2 \mathrm{~mm}$; the number of elements on the proximal femur models varied 
Table 1 The forces defined for walking and stair climbing

\begin{tabular}{|c|c|c|c|c|}
\hline & Force & $x$ & $y$ & $z$ \\
\hline \multirow[t]{2}{*}{ Walking } & Hip & -62.1 & -45.6 & -307.4 \\
\hline & Greater trochanter & 64.7 & 15.2 & 80.7 \\
\hline \multirow[t]{2}{*}{ Stair climbing } & Hip & -72.3 & -88.6 & -306.4 \\
\hline & Greater trochanter & 83 & 35.6 & 77.3 \\
\hline
\end{tabular}

from approximately 80,000 to 90,000 depending on the scan length. For all elements at the cortical/cancellous region, we calculated a bone volume fraction (BV/TV) and fabric tensor $\mathbf{M}$ as described earlier (Hazrati Marangalou et al. 2014a, 2012). In summary, for the elements that were at least partly within the cancellous bone, a spherical region around the element centroid with a diameter of $4 \mathrm{~mm}$ was defined (Harrigan et al. 1988) and the mean intercept length (MIL)-based fabric tensor was calculated for that spherical volume of interest using the image processing software provided by micro-CT system. For elements with a BV/TV $<0.02$, a reliable measurement of a fabric tensor is not possible and the identity tensor was used instead.

\subsection{Fabric estimation algorithm}

A total of three load cases were considered. The first load case represents walking, the second stair climbing and the third a combination of walking for $97 \%$ of the loading and stair climbing for $3 \%$ of the loading. The results of the latter will be discussed in detail, and the other load cases are merely provided to demonstrate the effect of using more simple loading conditions on the outcome of the fabric prediction. The magnitudes of the forces for walking and stair climbing (Table 1) were taken from previous studies (Bergmann et al. 2010; Heller et al. 2005).

Linear elastic analyses were performed for each individual load case (walking and stair climbing), and the stress tensors for each load case and each element were stored. The timeaveraged element stress tensor $\overline{\boldsymbol{\sigma}}$ then was calculated from the stress tensors stored for the two load cases $\sigma_{i}$ :

$\overline{\boldsymbol{\sigma}}=\sum_{i=1}^{2} \frac{n_{\text {cycles }, i}}{n_{\text {cylcles }, \text { tot }}} \sigma_{i}$

where $n_{\text {cycles, } i}$ relates to the number of cycles for that load case and $n_{\text {cycles, tot }}$ to the total number of cycles for all load cases. Eigenvectors of the averaged stress tensor were calcu- lated and used as an estimate of the fabric tensor. The eigenvalues of the average stress tensor were used to calculate the eigenvalues of the fabric tensor (Turner 1992). The eigenvalues of the stress tensor, however, were first scaled to be in agreement with the relationship between the normalized fabric tensor eigenvalues $\left(m_{i}\right)$, the bone volume fraction $(\rho)$ and the elastic modulus in the direction of the fabric tensor eigenvectors as proposed by Zysset and Curnier (1995):

$E_{i}=\varepsilon_{0} \rho^{k} m_{i}^{2 l}$

where $\varepsilon_{0}=8237.1 \mathrm{MPa}, k=2.5$ and $l=1.88$. This relationship thus involves a power of 3.76 for the relationship between fabric eigenvalues and Young's modulus. We assumed a similar relationship holds for the relationship between the eigenvalues of the stresses and fabric, and that the power can be rounded to 4 :

$\sigma_{\text {principal }, i}=\mathrm{cm}_{i}^{4}$

where $c$ is a constant. The eigenvalues of the fabric tensor then can be calculated from the inverse relationship. Since the fabric tensor is scaled such that $\operatorname{det}(\mathbf{M})=1 ;\left(\mathbf{M}=m_{i}\left(e_{i} \otimes\right.\right.$ $\left.e_{i}\right), e_{i}$ : eigenvectors $)$, the constant $c$ can be eliminated and the following relationship is obtained:

$m_{i}=\frac{1}{\left(\sigma_{\text {principal }, 1}^{1 / 4} \cdot \sigma_{\text {principal }, 2}^{1 / 4} \cdot \sigma_{\text {principal }, 3}^{1 / 4}\right)^{1 / 3}} \sigma_{\text {principal }, i}^{1 / 4}$

Figure 1 shows an overview of the iterative procedure. For the first iteration, no fabric estimate was available and the fabric tensor was set to the identity tensor, thus specifying isotropic material properties based only on the BV/TV distribution. The two load cases then were applied to the FE models, and the fabric tensor calculated. In the following iterations, orthotropic compliance tensors (Eq. 5) were assigned based on the fabric tensor calculated in the previous iteration (Zysset and Curnier 1995): 


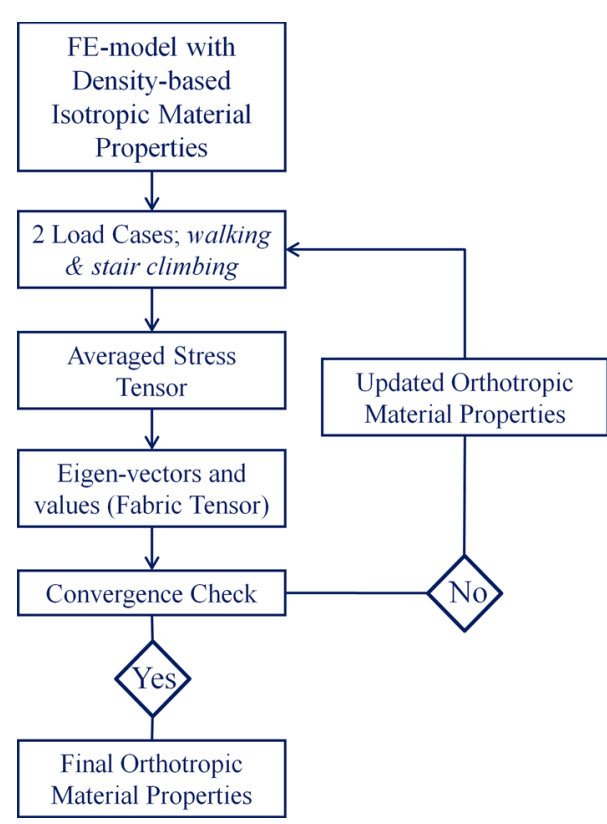

Fig. 1 Overview of the fabric estimation procedure

$$
\begin{aligned}
\mathbb{C}= & \sum_{i=1}^{3} \frac{1}{\varepsilon_{0} \rho^{k} m_{i}^{2 l}} M_{i} \otimes M_{i}-\sum_{i, j=1 ; i \neq j}^{3} \frac{v_{0}}{\varepsilon_{0} \rho^{k} m_{i}^{l} m_{j}^{l}} \\
& M_{i} \otimes M_{j}+\sum_{i, j=1 ; i \neq j}^{3} \frac{1}{2 G_{0} \rho^{k} m_{i}^{l} m_{j}^{l}} M_{i} \underline{\otimes} M_{j}
\end{aligned}
$$

where $v_{0}=0.3$ and $G_{0}=3168 \mathrm{MPa}$ are elastic constants, $m_{i}$ is the normalized eigenvalues, $M_{i}$ is the dyadic product of the eigenvectors of fabric tensor $\mathbf{M}$, and the powers $k$ and $l$ are as defined earlier. FE analyses were repeated, and a new estimate of the fabric tensor was calculated. Iterations continued until no further reduction was found in calculated average stress tensor.

\subsection{Accuracy check}

\subsubsection{Statistical analysis}

For the elements within the cancellous region, an elementwise Hotelling's $T^{2}$ test is used for the comparison of the eigenvalues and the eigenvectors of estimated and measured fabric tensors for the 10 samples used in this study (Schwartzman et al. 2010). This method is commonly used for group comparison of the eigenvalues and the eigenvectors of the diffusion tensors in magnetic resonance imaging (Lepore et al. 2008; Whitcher et al. 2007).

To test the differences in the eigenvalues and the eigenvectors of measured and estimated fabric tensors, two likelihood ratio tests (LRT) are calculated (Schwartzman et al. 2010). Cumulative distribution functions (CDF) of the eigenvalue and the eigenvector LRT values are approximated as the distribution of a scaled chi-squared variable, and the $p$ value is computed as $1-\mathrm{F}(\mathrm{LRT})$, where $\mathrm{F}$ is the cumulative distribution function of LRT values. Another cumulative distribution function is calculated for the $p$ values ( $\mathrm{F}(p$ value $)$ ) which describes the probability of finding a $p$ value less than a specific value. Low values of the CDF at low $p$ values indicate that the probability of getting a significant difference between measured and estimated fabric tensors is low, hence that the regions where significant differences are found are small. In contrast, a high values of CDF for low $p$ values indicate that differences between the measured and estimated fabric tensors are likely significant for large regions. Finally, contour plots of the $p$ value distribution for eigenvalues and eigenvectors are made.

For further interpretation of the results, the angular deviation between the estimated primary fabric direction and the measured one was calculated as a measure of eigenvector agreement. The agreement between the eigenvalues of the estimated and the measured fabric tensors was also quantified by the tensor norm error (Eq. 6).

err $=\frac{\left\|M_{\text {measured }}-M_{\text {estimated }}\right\|}{\left\|M_{\text {measured }}\right\|}$

\subsubsection{Finite element analyses}

In order to further test the effect of the estimated fabric tensor on the calculated mechanical properties, the results of models with material properties based on these estimated fabric tensors were compared to those of models generated in an earlier study (Hazrati Marangalou et al. 2013). In that earlier study, a reference model was generated for each bone in which orthotropic material properties were defined for elements within the cancellous region based on the actually measured fabric tensors and isotropic material properties were defined for the elements within cortical region while a rule of mixture was used for elements covering both compartments. As in that previous study, this model will serve as the gold standard relative to which the results of the model with estimated fabric are compared. The second reference model was an isotropic one with material properties based only on the density distribution. In the present study, we will also compare the results of the model with estimated fabric to those of these isotropic models.

For all models, elastic plastic damage constitutive behavior in local form (Charlebois et al. 2010) was used to simulate failure and post-failure behavior of the 10 femurs. Loading conditions represented a fall to the side configuration (Courtney et al. 1995; Verhulp et al. 2008). Whole-bone stiffness, maximum load and stress distribution of the orthotropic models based on estimated fabric were compared to the gold standard and isotropic models of the previous study. Differences were quantified by calculating root-mean-square errors 


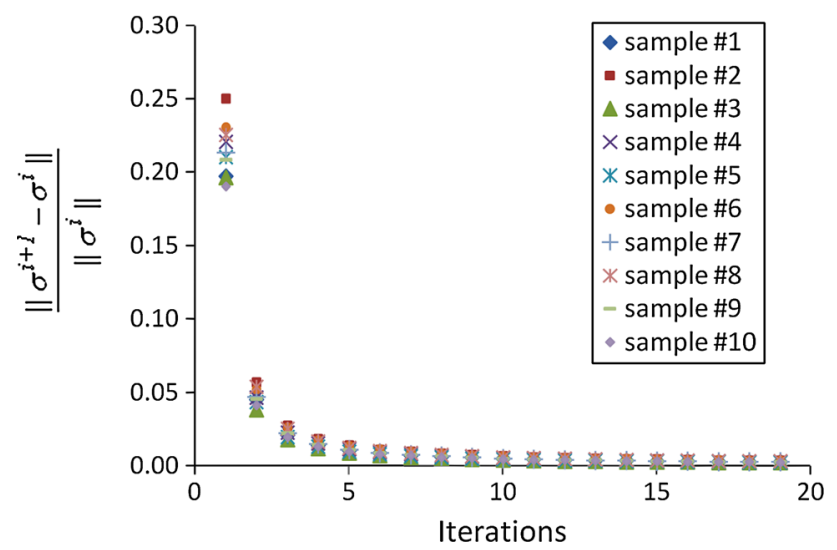

Fig. 2 Convergence checks between to sequential iterations

(RMSE), determination coefficients and concordance correlation coefficients. One-way ANOVA was used to test the significance of differences $(\mathrm{p}<0.05)$.

\section{Results}

The iterative procedure was continued until the norm error between sequential averaged stress tensors is below one percent (Fig. 2). Less than 20 iterations were needed to reach a converged state. Using 1 processor for the analyses, this required less than an hour per femur.

The averaged fabric tensor norm error as well as the average angular deviation relative to the gold standard was larger for the load cases that consist of only one load (load case 1 and 2) than for the combined load case (Table 2). This indicates that using multiple load cases can lead to better predictions of the fabric direction. In the remainder of this section, thus only results for the third (combined) load case will be presented.

From the vector plot of the primary fabric direction, it can be seen that the estimated fabric primary directions generally agree well with those based on micro-CT measurements (Fig. 3). In some regions, however, large deviations between estimated and measured vectors were found particularly in Ward's triangle region and the shaft region, which are lowdensity regions. This, however, is due to the fact that in the plot of the measured fabric the identity tensor was assumed for elements with a BV/TV $<0.02$, whereas in the plot of the estimated fabric tensors the actual estimated values were plotted. When averaged over all elements within the cancellous region, the angular deviation between the estimated primary fabric direction and the measured one was $32.8^{\circ}$. For this calculation, the elements with a $\mathrm{BV} / \mathrm{TV}<0.02$, for which an isotropic fabric tensor was specified, were not included.

Figure 4 shows the distribution of the estimated $p$ values for the eigenvalue and eigenvector tests. For most elements, the $p$ values calculated for the eigenvalues and eigenvectors were larger than 0.05 , indicating that no significant differences could be detected between the estimated and measured fabric distribution. Contour plots of $p$ value distributions show that high $p$ values usually occur in the cancellous region, while lower $p$ values are in the cortical regions (Fig. 5). The latter is due to the fact that for cortical bone an isotropic fabric tensor was defined in the measured model, leading to an arbitrary orientation of the eigenvectors, whereas the three eigenvalues per element now are equal. Furthermore, significant differences in the eigenvalues and the eigenvectors are found at the location where the external forces were applied. At these locations, relatively large deformations are found, resulting in some element distortion, which in turn leads to disagreement in the eigenvalues and the eigenvectors.

The averaged norm difference between the actual and the estimated fabric tensors varied between 8.19 and $9.75 \%$ for the ten samples (mean $=9.21 \%, n=10)$. However, when comparing the degree of anisotropy, only a very low correlation was found between the estimated and measured values, with an average coefficient of determination of $R^{2}=0.11$.

Contour plots of the von-Mises stress and the damage distribution are shown in Fig. 6. For comparison, results of the gold standard model and the isotropic model as presented in an earlier publication are reproduced in this figure as well (Hazrati Marangalou et al. 2013). The results show that the orthotropic model with estimated fabric tensors tends to slightly overestimate the stresses. Although there are differences in the stress magnitude between the models, the distributions of the stresses in all models are rather similar. When plotting the amount of damage, the orthotropic models with
Table 2 Effect of adding more load cases in fabric estimation algorithm

\begin{tabular}{llll}
\hline & Load case 1 & Load case 2 & Load case 3 \\
\hline Walking $(\%)$ & 100 & 0 & 97 \\
Stair climbing (\%) & 0 & 100 & 3 \\
$\begin{array}{c}\text { Averaged fabric tensor norm error } \\
\text { relative to gold standard (\%) }\end{array}$ & $9.67(8.68-10.50)$ & $9.74(8.80-10.59)$ & $9.21(8.19-9.75)$ \\
$\begin{array}{c}\text { Averaged angular deviation } \\
\text { relative to gold standard }\left({ }^{\circ}\right)\end{array}$ & $39.4(21.7-48.2)$ & $39.3(21.4-47.6)$ & $32.8(24.4-40.1)$ \\
\hline
\end{tabular}




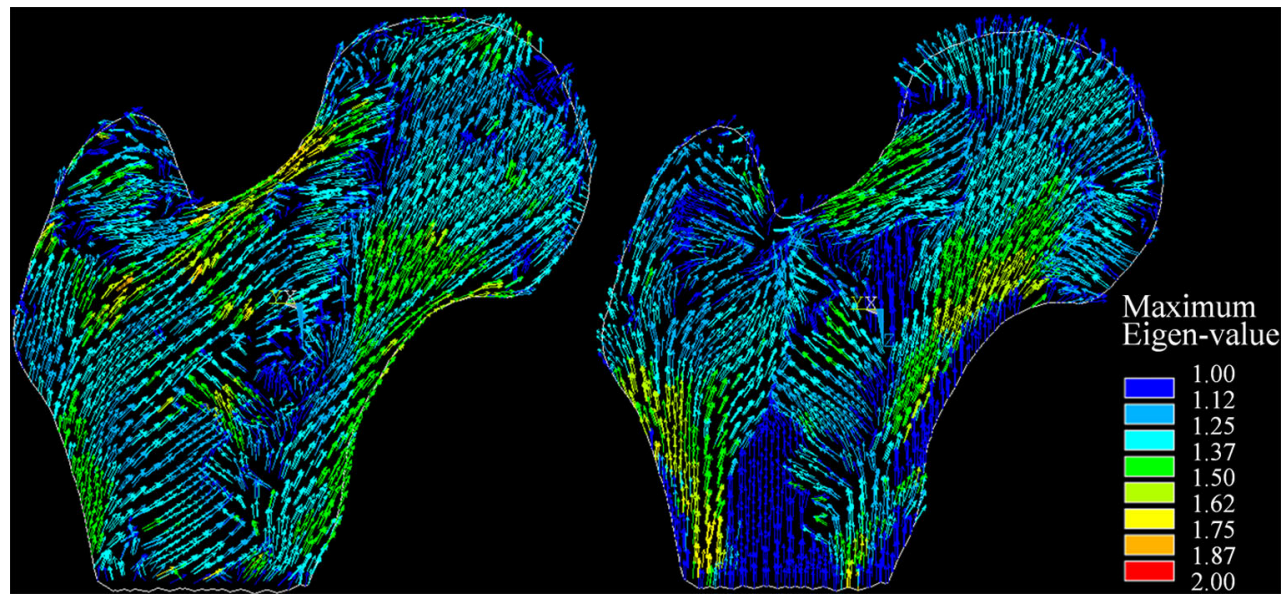

Fig. 3 Vector plot of the estimated (left) and measured (right) major fabric direction, vector colors show the maximum eigenvalue of fabric tensor

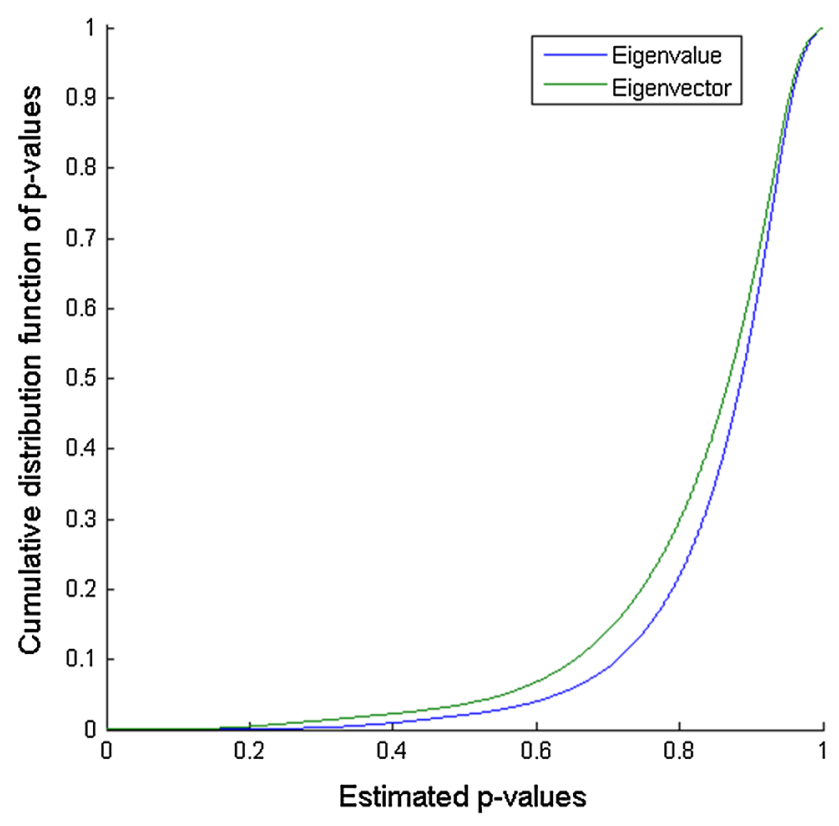

Fig. 4 Cumulative distribution function of estimated $p$ values from eigenvalue and eigenvector tests $(n=10)$

estimated fabric tensors underestimate the amount of damage at the femoral neck and produce a distribution that is more similar to that of the isotropic model.

Whole-bone force displacement curves show that the orthotropic model with estimated fabric tensors can well replicate the force-displacement curves found for the gold standard and behaves better than the isotropic model that underestimates both the stiffness and strength (Fig. 7). For the orthotropic model with estimated fabric, the root-meansquare error (RMSE) for whole-bone stiffness, taking the gold standard as the reference, was $11.8 \%$, which is less than the error found earlier for the isotropic model $(26.3 \%)$. A good correlation was found for whole-bone stiffness with determination coefficient of $89.2 \%$ and concordance correlation (CC) of $87 \%$. For the ultimate load, an RMSE of $8.4 \%$ was found for orthotropic models with estimated fabric, when compared to the gold standard, which again was less than the error found earlier for the isotropic model (14.5\%). Similar to the stiffness, good correlations were found with an $R^{2}=97.2 \%$ and $\mathrm{CC}=92.6 \%$ (Fig. 8).

Results of the ANOVA test revealed that no significant differences exist between the results of the orthotropic models with estimated fabric and the gold standard, neither for whole-bone properties nor for the stress/damage distributions.

\section{Discussion}

The first goal of this study was to investigate the feasibility and accuracy of an iterative approach to calculate the fabric tensor from the principal stress distribution. We found that, indeed, this approach can lead to reasonable estimates of the fabric tensor orientation. On average, the difference in norm between estimated fabric tensors and the fabric tensors measured from the bone micro-architecture was less than $10 \%$. However, the degree of anisotropy of the estimated and the measured fabric tensors did not compare well, and at some locations, the orientations of the estimated fabric tensor deviated much from that of the measured ones. The reason for this seemingly discrepancy is that the tensor norm is largely determined by the largest components of the tensor, whereas the degree of anisotropy is largely determined by the smallest tensor component. The estimation algorithm used here can predict the primary fabric direction fairly well, but the prediction of the secondary and tertiary directions and values will be much less accurate. Because the primary direction is well predicted, the stress calculation of the FE model with its anisotropy based on estimated fabric compared very well 


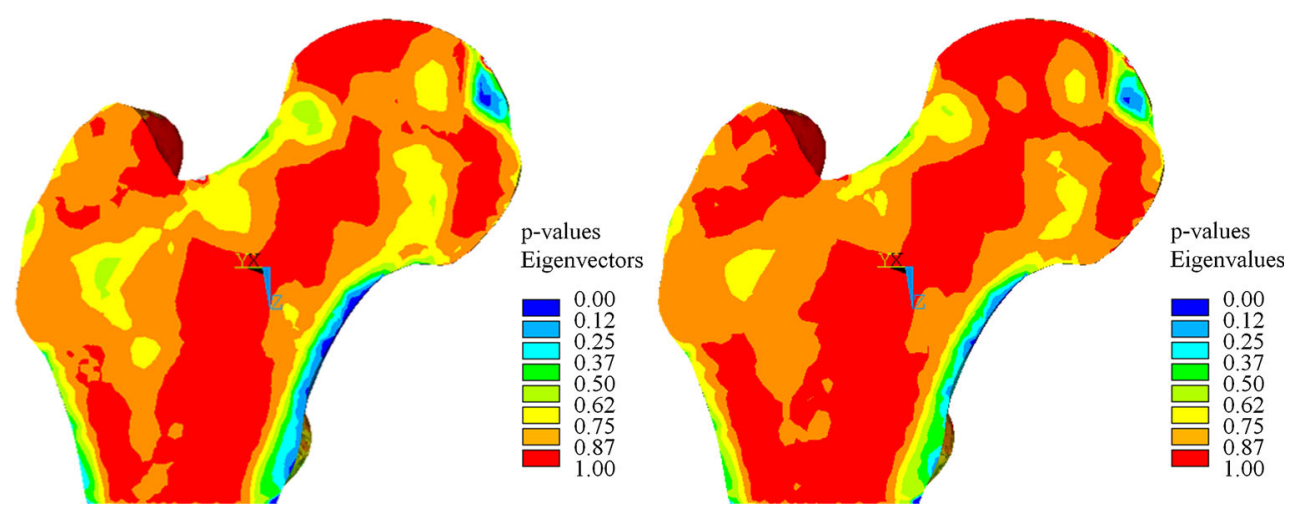

Fig. 5 Contour plots of $p$ value distribution for eignvectors (left) and eigenvalues (right)
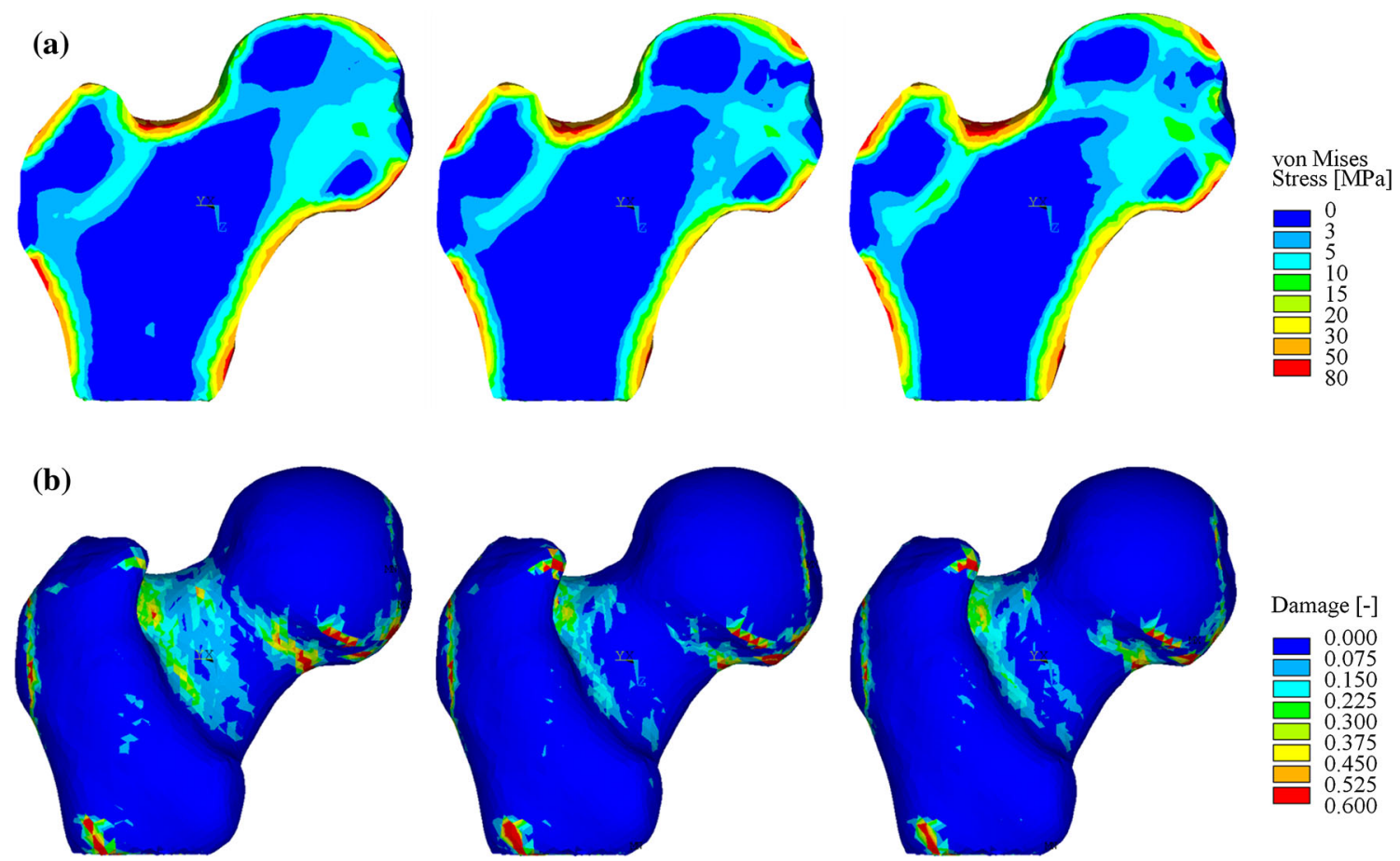

Fig. 6 a von-Mises stress and $\mathbf{b}$ damage parameter distribution in the gold standard (left), isotropic (middle) and orthotropic model with estimated fabric (right). Results for the gold standard model and the isotropic model are reproduced from an earlier publication. (Hazrati Marangalou et al. 2013)

to that of a FE model with its anisotropy based on measured fabric. In regions where the correspondence was not very good, the stresses are either low or the material anisotropy is not very pronounced.

The second goal of this study was to investigate whether such micro-structural enhanced orthotropic models based on estimated fabric can provide more accurate results than a database approach introduced earlier or than using isotropic models (Hazrati Marangalou et al. 2013). In that earlier study, we found that, compared with the gold standard, using database-derived models resulted in a RMS error of 4.9 and $3.1 \%$ for the stiffness and ultimate load, respectively. In the present study, using the estimated fabric, these errors were 11.8 and $8.4 \%$, respectively, indicating that the approach tested in the present study is less accurate than the database approach. For the standard isotropic model investigated in that earlier study (named isotropic-I), these errors were 26.3 and $14.5 \%$, respectively, demonstrating that the orthotropic models based on estimated fabric as tested here can produce more accurate results than the isotropic model. As was indicated in the earlier study, significant differences were found for bone stiffness, the stress and damage distribution, when comparing results obtained from the isotropic model with the gold standard, whereas no significant differences were found 


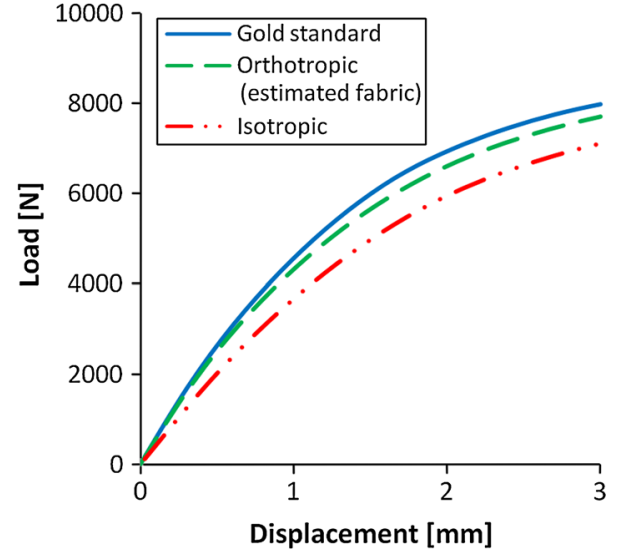

Fig. 7 Typical force-displacement curves obtained from different orthotropic and isotropic models (adapted and extended from Hazrati Marangalou et al. 2013)

for results obtained from the orthotropic model based on estimated fabric as tested here. It should be noted, though, that differences between the isotropic model and the gold standard, though significant, are generally small.

The approach used here bears some resemblance with that used in earlier bone remodeling simulation studies (Beaupré et al. 1990; Jacobs et al. 1997). In these earlier studies, however, the evolution of bone density and anisotropy was simulated, starting from a homogeneous and isotropic situation. The approach used here differs from this approach in the sense that we do not aim at predicting the evolution of density and anisotropy over time, but rather try to back calculate the anisotropy for a given loading and fixed density distribution. As such, the approach presented here should not be considered a bone remodeling simulation but rather an optimization procedure. Differences also exist in the way the bone anisotropy was calculated. In the earlier studies, the

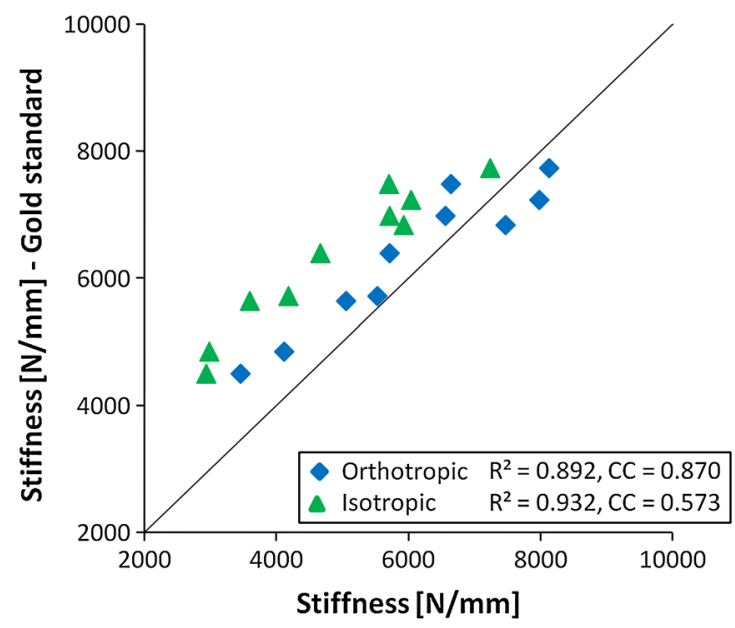

Fig. 8 Correlation of results with those obtained for the gold standard for whole-bone stiffness (left) and ultimate load (right) when implementing isotropic material properties or anisotropic properties based anisotropic compliance matrix was directly calculated from the stress tensor, whereas in our approach the fabric tensor is calculated from the stress tensor, and empirical laws are used to derive the compliance matrix. This has the advantage that we can compare the results directly with morphology measurements and that we can use previously validated material models.

It is possible that the accuracy of the present approach may be further improved by analyzing more load cases to better represent the state of loading toward which the bones are adapted. In the present study, we only investigated the effect of two loading modes (walking and stair climbing). It is interesting to see that a combination of both loading modes provides better estimates of the bone fabric direction than a single one. Applying a more realistic daily loading history would be straightforward, but since no information about the donors' history was available this was not done here. It is thus possible that the loading history applied here does not represent that of the donor very well. This could lead to differences between the estimated and measured fabric directions. Therefore, we expect that subject-specific models that account for parameters such as subject-specific loading magnitudes and muscle attachment sites may improve the results. A possible approach to obtain such information could be the use of newly developed load estimation algorithms to define the loading history to which the bones are adapted (Campoli et al. 2012; Christen et al. 2012). This could also make the approach developed here suitable for bones at different sites for which no information about the loading history is available. Nevertheless, it is promising that even when using only a few generic load cases as done in the present study, reasonable results were obtained.

The results demonstrate that only a few iterations are needed to reach a converged state and that further iterations

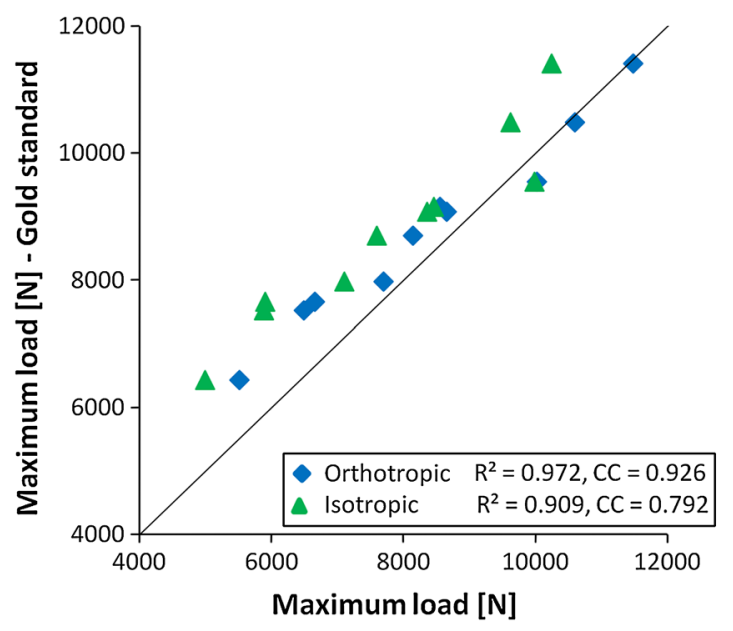

on an estimated fabric tensor. Results for the isotropic and gold standard models are reproduced from Hazrati Marangalou et al. (2013) 
will not further improve the results. The fact that the angular deviation between estimated and measured primary fabric direction is very large $\left(\sim 90^{\circ}\right)$ in some regions (Figure 3 ) is probably due to the fact that, in particular for near isotropic stress states, the principal directions can change order. In such cases, a small change in magnitude can result in a 90 degrees change in the principal directions.

Some limitations of this study must be mentioned as well. First, only a limited number of bones were tested, and since these were obtained from an elderly population, it is not known whether similar results would be obtained for younger subjects. Nevertheless, the age group investigated here is relevant for one of the main applications of the tested approach, being improving preclinical testing of bone-implant configurations. Second, to be able to create a gold standard model with measured fabric, we used micro-CT images for the generation of the continuum FE models. For clinical application, however, clinical CT will need to be used. Nevertheless, since bone geometry and density can be measured accurately from clinical CT scans, we expect that similar accurate results can be obtained when using such scans. It should be noted here that the approach used here is rather insensitive for the image resolution. Since bone density is homogenized over a 4-mm spherical region, the resolution of any clinical CT should suffice for determination of the density distribution. Third, in this study, the cortical bone was always assumed isotropic. Although it would be possible to define orthotropic properties for the cortical bone as well using the fabric estimation algorithm, we expect that this will not affect the results presented here too much since for the loading configurations used here the cortical bone is merely loaded in the longitudinal direction (by bending or compression), such that only the stiffness in that direction is relevant.

In conclusion, we have demonstrated that this novel approach can be used to estimate the fabric tensor of the proximal femur with some fair accuracy. This procedure could lead to the generation of FE models that can account for bone anisotropic material properties. Although the results were slightly less accurate than those of a database approach developed earlier, a major advantage of the present approach is that it does not require such a database and can be applied to any bone for which reasonable estimates of the loading history are available. We expect that this approach can lead to more accurate results in particular for models used to study implants, which are usually anchored in highly anisotropic cancellous bone regions.

Acknowledgments Funding from the European Union for the osteoporotic virtual physiological human project (VPHOP FP7-ICT2008223865) is gratefully acknowledged.

Conflict of interest Bert van Rietbergen is a consultant for Scanco Medical AG.

\section{References}

Beaupré GS, Orr TE, Carter DR (1990) An approach for time-dependent bone modeling and remodeling-theoretical development. J Orthop Res 8:651-661

Bergmann G, Graichen F, Rohlmann A, Bender A, Heinlein B, Duda GN, Heller MO, Morlock MM (2010) Realistic loads for testing hip implants. Bio-Med Mater Eng 20:65-75

Campoli G, Weinans H, Zadpoor AA (2012) Computational load estimation of the femur. J Mech Beh Biomed Mater 10:108-119

Carter DR, Hayes WC (1976) Bone compressive strength: the influence of density and strain rate. Science 194:1174-1176

Charlebois M, Jirasek M, Zysset PK (2010) A nonlocal constitutive model for trabecular bone softening in compression. Biomech Model Mechanobiol 9:597-611

Christen P, van Rietbergen B, Lambers F, Müller R, Ito K (2012) Bone morphology allows estimation of loading history in a murine model of bone adaptation. Biomech Model Mechanobiol 11:483-492

Courtney AC, Wachtel EF, Myers ER, Hayes WC (1995) Age-related reductions in the strength of the femur tested in a fall-loading configuration. J Bone Joint Surg Am 77:387-395

Cowin SC (1985) The relationship between the elasticity tensor and the fabric tensor. Mech Mater 4:137-147

Fyhrie DP, Carter DR (1986) A unifying principle relating stress to trabecular bone morphology. J Orthop Res 4:304-317

Harrigan TP, Mann RW (1984) Characterization of microstructural anisotropy in orthotropic materials using a second rank tensor. J Mater Sci 19:761-767

Harrigan TP, Jasty M, Mann RW, Harris WH (1988) Limitations of the continuum assumption in cancellous bone. J Biomech 21:269-275

Hazrati Marangalou J, Ito K, van Rietbergen B (2012) A new approach to determine the accuracy of morphology-elasticity relationships in continuum FE analyses of human proximal femur. J Biomech 45:2884-2892

Hazrati Marangalou J, Ito K, Cataldi M, Taddei F, van Rietbergen B (2013) A novel approach to estimate trabecular bone anisotropy using a database approach. J Biomech 46:2356-2362

Hazrati Marangalou J, Eckstein F, Kuhn V, Ito K, Cataldi M, Taddei F, Rietbergen B (2014a) Locally measured microstructural parameters are better associated with vertebral strength than whole bone density. Osteoporos Int 25:1285-1296

Hazrati Marangalou J, Ito K, Taddei F, van Rietbergen B (2014b) Interindividual variability of bone density and morphology distribution in the proximal femur and T12 vertebra. Bone 60:213-220

Heller MO, Bergmann G, Kassi JP, Claes L, Haas NP, Duda GN (2005) Determination of muscle loading at the hip joint for use in pre-clinical testing. J Biomech 38:1155-1163

Jacobs CR, Simo JC, Beaupre GS, Carter DR (1997) Adaptive bone remodeling incorporating simultaneous density and anisotropy considerations. J Biomech 30:603-613

Keyak JH (2001) Improved prediction of proximal femoral fracture load using nonlinear finite element models. Med Eng Phys 23:165-173

Lepore F, Brun C, Yi-Yu C, Ming-Chang C, Dutton RA, Hayashi KM, Luders E, Lopez OL, Aizenstein HJ, Toga AW, Becker JT, Thompson PM (2008) Generalized tensor-based morphometry of HIV/AIDS using multivariate statistics on deformation tensors. IEEE Trans Med Imaging 27:129-141

Morgan EF, Bayraktar HH, Keaveny TM (2003) Trabecular bone modulus-density relationships depend on anatomic site. J Biomech 36:897-904

Odgaard A (1997) Three-dimensional methods for quantification of cancellous bone architecture. Bone 20:315-328

Odgaard A, Kabel J, van Rietbergen B, Dalstra M, Huiskes R (1997) Fabric and elastic principal directions of cancellous bone are closely related. J Biomech 30:487-495 
Pahr DH, Zysset PK (2009) A comparison of enhanced continuum FE with micro FE models of human vertebral bodies. J Biomech 42: 455-462

Rice JC, Cowin SC, Bowman JA (1988) On the dependence of the elasticity and strength of cancellous bone on apparent density. J Biomech 21:155-168

Schwartzman A, Dougherty RF, Taylor JE (2010) Group comparison of eigenvalues and eigenvectors of diffusion tensors. J Am Stat Assoc 105:588-599

Taddei F, Schileo E, Helgason B, Cristofolini L, Viceconti M (2007) The material mapping strategy influences the accuracy of CT-based finite element models of bones: an evaluation against experimental measurements. Med Eng Phys 29:973-979

Turner CH (1992) On Wolff's law of trabecular architecture. J Biomech 25:1-9

Vander Sloten J, Van der Perre G (1989) Trabecular structure compared to stress trajectories in the proximal femur and the calcaneus. J Biomed Eng 11:203-208
Verhulp E, van Rietbergen B, Huiskes R (2008) Load distribution in the healthy and osteoporotic human proximal femur during a fall to the side. Bone 42:30-35

Whitcher B, Wisco JJ, Hadjikhani N, Tuch DS (2007) Statistical group comparison of diffusion tensors via multivariate hypothesis testing. Magn Reson Med 57:1065-1074

Yang G, Kabel J, Van Rietbergen B, Odgaard A, Huiskes R, Cown S (1998) The anisotropic Hooke's law for cancellous bone and wood. J Elast 53:125-146

Zysset PK, Curnier A (1995) An alternative model for anisotropic elasticity based on fabric tensors. Mech Mater 21:243-250

Zysset PK, Goulet RW, Hollister SJ (1998) A global relationship between trabecular bone morphology and homogenized elastic properties. J Biomech Eng 120:640-646 